\title{
LA MÉTHOdE SYNTHÉTIQUE DANS L'ÉTUDE DU LAIT. LE LAIT AU POINT DE VUE COLLOIDAL. RECHERCHES SUR LE MÉCANISME DE L'ACTION DE LA PRÉSURE
}

\author{
par Ch. PORCHER,
}

Docteur ès sciences physiques

(Suite)

\section{LA TEMPÉRATURE OPTIMUM DE L'ACTION DE LA PRÉSURE}

La présure de caillette de veau résiste aux basses températures, puisqu'elle a pu être portée à - $180^{\circ}$ sans être touchée ; mais la chaleur l'altère. Hammarsten estimait déjà que la température de $37^{\circ}$ nuisait par son action prolongée. Ce sont les températures supérieures qui sont les plus nocives, lorsque la présure est en solution aqueuse. Lorsqu'elle est à l'état de poudre extrêmement sèche, elle peut être portée jusqu'à $130^{\circ}$ sans altération sensible. En solution, la présure résiste d'autant mieux qu'elle est plus concentrée. LöRCHER a montré que ce sont les extraits glycérinés qui supportent mieux les températures les plus élevées. Enfin, ce qui est plus intéressant encore à considérer, e'est de déterminer la température optimum d'action de la présure.

Déjà Storch et SegeLCKe avaient indiqué la température de 41025. Fleischmann (232) a étudié également l'action de la présure entre $15^{\circ}$ et $50^{\circ}$, et bien que ses expériences soient peu nombreuses, il semble en résulter que la marche du phénomène puisse se traduire par une courbe logarithmique. C'est à cette conclusion que sont arrivés plus tard Vieth et Siegreld. Pour Grimmer et Kruger, $40^{\circ}$ est la température optimum. FuLD portait cet optimum à $45^{\circ}$.

La courbe que nous donnons résume des recherches répétées et qui sont d'accord avec celles de GRTMMER et KRÜGER; la température optimum d'action de la présure est done bien de $40^{\circ}$. Les temps de coagulation représentent une fonction logarithmique de la température.

L'acidité du lait frais était de $17^{\circ} \mathrm{D}$. La force de la présure était telle que, à $40^{\circ}$ avec $25 \mathrm{~cm}^{3}$ de lait et $1 / 2 \mathrm{~cm}^{3}$ de présure, il y avait prise en $345 \mathrm{sec}$.

On a fait agir la même présure sur la même quantité de lait. On élève la température de ce dernier de $5^{\circ}$ en $5^{\circ}$ depuis $15^{\circ}$ jusqu'à $50^{\circ}$ et voici les résultats trouvés :

(232) Fuischmann. - Das Molkereiwesen, 1876, p. 745. 


\section{TABLEAU XL.}

\section{TEMPS DE PRISE DU LAIT EN FONCTION DE LA TEMPERATURE,}

Temps de prise

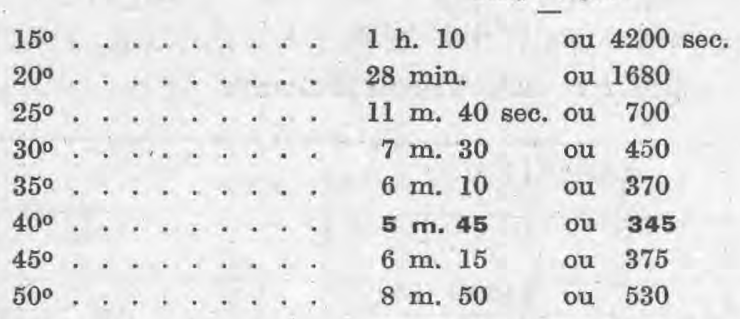

Il y a un minimum qui correspond à $40^{\circ}$; c'est la température pour laquelle la présure a son maximum d'action. On pourrait penser que la détermination de la force de la présure, au lieu de s'effectuer à $35^{\circ}$ comme il est dit dans les ouvrages, devrait se faire à $40^{\circ}$, température optima ; mais c'est que dans l'industrie, la température de $35^{\circ}$, - encore est-ce 'un grand maximum, - est plus communément suivie pour toutes les espèces de fromages que celle de $40^{\circ}$ qui n'est qu'exceptionnellement notée.

On doit se demander aussi quelle est la température minimum à laquelle la présure cesse d'agir.

On l'estimait autrefois à 15o. Mais L. Camus et L. GLeY (233), Morgenroth, Fuld, ont montré qu'à $8^{\circ}$ et au-dessous, la présure agit encore à la condition qu'on laisse les liqueurs pendant très longtemps à cette température, au moins 24 à 48 heures. La preuve en est donnée par le fait que du lait emprésuré à froid coagule rapidement quand on le porte à $32^{\circ}$.

L'acte essentiel de l'emprésurage est dans le dédoublement de la caséine en paracaséine et une protéose soluble. Pourquoi sous l'action des basses températures, la coagulation qui est la traduction visible du second temps de l'emprésurage ne s'effectue-t-elle pas ? Plus on fait agir la présure à une température élevée, plus facilement et plus rapidement le paracaséinate de calcium précipite ; au contraire, plus on refroidit, plus il faut de sels calciques pour amener la même précipitation.

Nous pensons également que les phénomènes de protection sont plus marqués aux basses températures qu'aux températures hautes, et si l'attaque de la caséine se fait très lentement aux basses températures, la protection du paracaséinate par le caséinate non encore attaqué, doit être très manifeste.

Dans l'action de la chaleur sur la présure, il est indiqué d'examiner l'influence de la réaction de la liqueur.

(233) L. Camos et L. Glex. - Persistance d'activité de la présure à des températures basses ou élevées. C. R. Ac. Sc. 1897, 125, 256. 
Nous avons vu, en effet, que si la présure agit dans un milieu vraiment neutre, c'est-à-dire de $p H=7$ ou même $>7$, et allant jusqu'à

\section{GRAPHIQUE IV}

LA TEMPÉRATURE OPTIMUM D'AGTION DE LA PRÉSURE

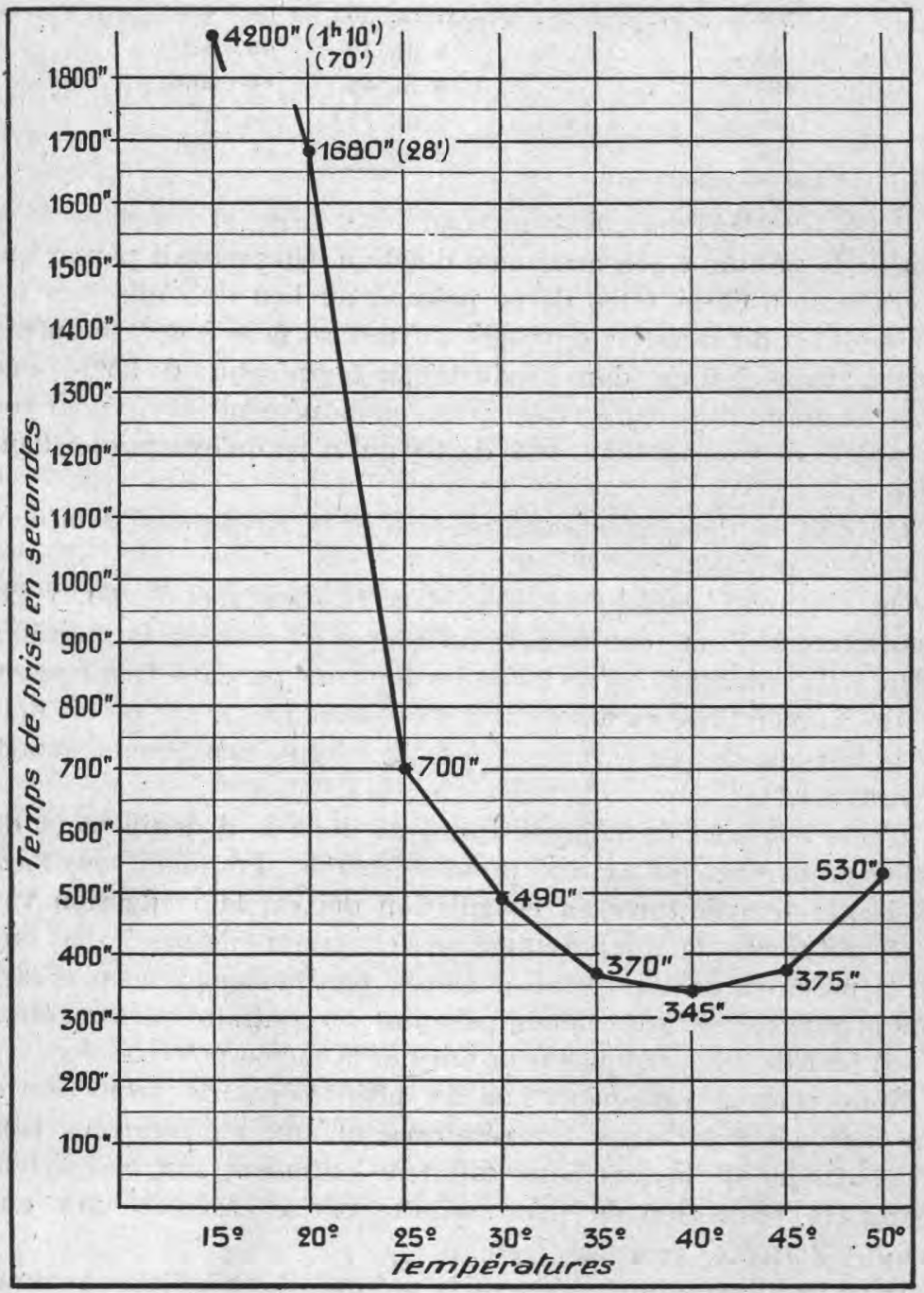

7,4-7,5, son action n'en est pas moins très facilitée par les acides. C'est l'influence de ceux-ci que nous allons d'abord étudier et nous la feront suivre de celle des alcalins. 


\section{INFLUENCE DE L'ACIDITE SUR L'EMPRÉSURAGE}

Lorsqu'on cherche à déterminer la force de certaines solutions de présure, on est amené à constater que pour des quantités de lait et de présure toujours les mêmes, on peut obtenir des temps de coagulation très différents, et par suite, des produits différents. C'est qu'un nouveau facteur est intervenu : l'acidité du lait, ou même l'acidité de la présure. ainsi qu'on le verra un peu plus loin en dépouillant le travail de GRTMMER et RUDZIK (234).

Storch et Segelcke, dans leurs recherches sur l'action de la présure et la possibilité de la mettre en équation, avaient déjà remarqué combien grande était l'importance du facteur acidité, d'où la nécessité de le faire entrer dans l'étude mathématique de l'action de la présure.

AUFSBERG (235) divise en deux parties le lait à mettre en expériences, l'une est conservée à basse température et l'autre portée à $30^{\circ}$ environ pour qu'elle s'acidifie rapidement; puis, il mélange des proportions diverses des deux liqueurs de façon à réaliser des échantillons de lait dont l'acidité croît au fur et à mesure que la proportion du lait acide s'élève de son côté. Il note qu'une augmentation d'acidité de $1^{\circ}$ se fait sentir surtout pour les premières acidités, c'est-à-dire au début; autrement dit, l'acidité agit d'autant plus fortement que l'on part de l'acidité normale du lait. C'est ce qui se trouvera d'ailleurs traduit dans les courbes que nous avons établies à l'aide de nos recherches personnelles.

GRTMMER et KRÜGER (226) donnent aux recherches d'AuFsBerG une traduction mathématique et montrent que les temps de coagulation sont une fonction exponentielle du degré d'acidité.

Laisser le lait s'acidifier, comme l'a fait AJFsBERg, n'est peutêtre pas toujours un processus heureux, parce que nous ignorons tout de la flore du lait mis en expérience, et il peut se faire, - ceci n'est pas une simple vue de l'esprit, mais s'appuie sur les constatations de tous ceux qui travaillent sur le lait, - que la flore composite dont le développement engendre l'hyperacidité du lait renferme des espèces présurigènes. Il suffit de noter ce fait pour comprendre le trouble apporté dans les expériences.

Nous préférons 'de beaucoup la technique de Alleman et ScHmidT (228) qui ajoutent au lait des quantités déterminées d'acide acétique.

Vieth et Stegreld (236) plus logiquement ont recours à l'acide lactique, de la fermentation spontanée du lait, mais leurs observations, comme celles de Alueman et Schmidt (228), de Grimmer et KrüGger

(234) W. Grimmer et W. Runzick. - Beiträge zur Kenntnis der Labwirkung. $2^{e}$ mémoire. - Milch. Forsch. 1926, 3, 361 .

(235) TH. Aursberg. - Sâuregrad der Miloh und Labwirkung. Molkerei Zeitung Bildesheim, 1900, 14, 293.

(236) P. Vieth et M. StEGFed, - Ueber Labwirkung und Labprüfung. Milchzeitung, $1900,29,657$ et 673 . 
(224), conduisent à mettre en relief cette donnée que les vitesses de coagulation représentent une fonction logarithmique de l'acidité. Mais il ne s'agit dans toutes ces recherches que d'acidité évaluée titrimétriquement.

Aujourd'hui que l'on peut déterminer l'acidité en fonction du nombre des ions $H$, il est intéressant de superposer aux données titrimétriques les données potentiométriques, car, - et en cela tous les auteurs sont d'accord, - il est préférable de recourir à la méthode électrique, l'emploi des indicateurs colorés ayant une moindre sensibilité.

Il est évident, d'après les recherches de tous, ainsi que des nôtres propres, que l'acidité du lait est un facteur beaucoup plus important qu'on ne l'avait cru jusqu'ici. Aussi, de toute nécessité, doit-on, dans le calcul de la force de la présure, bien spécifier en présence de quelle acidité on se trouve.

Ma:s s'il est facile de dégager cette remarque eapitale, il faut, par contre, bien spécifier que l'indication de l'acidité ne vaut que si on la place dans une suite de recherches de même ordre et très voisines dans le temps, car une même valeur de cette donnée ne correspond pas nécessairement à des laits identiques.

Nous avons eu l'oceasion d'appeler l'attention sur l'importance de l'étalonnage du lait dans les recherches sur la présure, et de montrer que pour des expériences s'étalant sur une longue série de jours, il fallait être assuré d'avoir un lait qui fût toujours semblable à lui-même.

Dans l'industrie laitière, on a toujours affaire à des laits de grands mélanges dont le type varie peu du jour au lendemain dans une même exploitation, et l'on paut dire qu'ils sont assez semblables d'un jour à l'autre. Aussi, les recherches que les auteurs peuvent faire en utilisant de tels laits comportent des éléments de comparaison intéressants ; e'est le eas de Mundiger (237). Non seulement, ces laits varient peu du jour au lendemain, dans une même exploitation ou dans une même laiterie, au point de vue chimique, mais également fort peu dans leur façon de réagir vis-à-vis de la présure.

Mais pour les recherches de laboratoire, on n'a toujours pas sous la main ces laits de grands mélanges, et nous devons nous contenter de laits individuels ou, tout au moins, de laits de petits mélanges provenant de deux ou trois animaux que l'on peut avoir à sa disposition.

LENK, pour vérifier la loi de STORCH, avait recours au lait qui provenait de la même vache traite le matin, et ce lait était conservé à la glacière jusqu'au moment où les essais étaient effectués dans l'aprèsmidi du même jour; il s'agissait donc ici de laits individuels. Or, ces laits manquent d'uniformité ; indiscutablement au point de vue de leur

(237) Mundiger. - Contribution à la conne.issance de l'action de la présure, Milch. Forsch. Mai 1927, 4. 368-390. 
taux butyreux, moins au point de vue de la grossière composition chimique de leur extrait dégraissé telle que l'analyse courante peut nous la donner, mais ils en manquent aussi au point de vue de leur réaction vis-à-vis de la présure et ils ne coagulent pas dans le même temps, avec la même quantité de la même prósure d'un jour à l'autre; il $\mathrm{y}$ a des écarts qui sont parfois assez sensibles.

Nous ne faisons pas allusion ici à l'emploi de laits individuels qui ne seraient pas tout à fait normaux, parce qu'ils proviennent de mamelles plus ou moins enflammées et qu'ils répondent au type des laits dits "paresseux"; nous restons dans le cadre des laits sains.

II n'est donc pas possible de définir un lait, considéré dans sa réaction vis-à-vis la présure, en lui attribuant un coefficient qui puisse être comparé à un coefficient du même ordre pour un autre lait. Il ne saurait y avoir de lait-étalon, dès l'instant où on a la prétention de s'adresser à du lait liquide frais. On a toute chance de réussir si l'on utilise, comme nous l'avons fait remarquer plus haut, du lait maigre reconstitué avec une poudre préparée selon le procédé Krause, lequel lait peut être additionné d'une quantité déterminée de $\mathrm{CaCl}^{2}$. Il est, en effet, plus que probable que les livraisons de poudres de lait qui se succèdent se ressemblent beaucoup dans leur façon de se comporter vis-à-vis de la présure, mais, cependant, on ne saurait identifier tout à fait deux livraisons qui sont fournies à plusieurs mois d'intervalle, car, entre les laits d'hiver et les laits d'été, il est des différences d'ordre chimique qui retentissent sur l'emprésurage.

Le véritable étalon pour apprécier une présure eonsiste dans l'obtention d'un système formé de caséinate de calcium + un sel soluble de calcium, lequel système serait toujours préparé dans les mêmes conditions de température, avee les mêmes masses de caséine, de chaux et du sel de calcium employé. En l'espèce, c'est le $\mathrm{CaCl}^{2}$ que nous recommandons; nous en verrons plus tard la raison.

Les divers facteurs de l'acidité du lait, - L'acidité du lait, comme nous l'avons fait ressortir, qu'elle soit déterminée titrimétriquement ou potentiométriquement, relève de facteurs différents, si bien qu'à une même acidité, soit titrimétrique, soit potentiométrique, ne correspond pas toujours le même équilibre salin du lait.

Si nous comparons différents laits, nous voyons, en effet, que leur degré d'acidité et leur $p \mathrm{H}$ ne correspondent pas toujours, autrement dit, à des degrès titrimétriques identiques ne répondent pas nécessairement des $p \mathrm{H}$ semblables et inversement.

Dans son travail, MuNDIGER estime qu'on ne saurait dire a priori que des laits de même $p \mathrm{H}$ doivent donner des temps de coagulation identiques, 
et la meilleure preuve, il l'emprunte à un tableau du travail de ALLEMANN (238).

\section{TABLEAU XLI}

AGIDITÉ 'TITRIMÉTRIQUE, GONGENTRATION EN IONS H ET VITESSE DE GOAGULATION DE LAITS INDIVIDUELS.

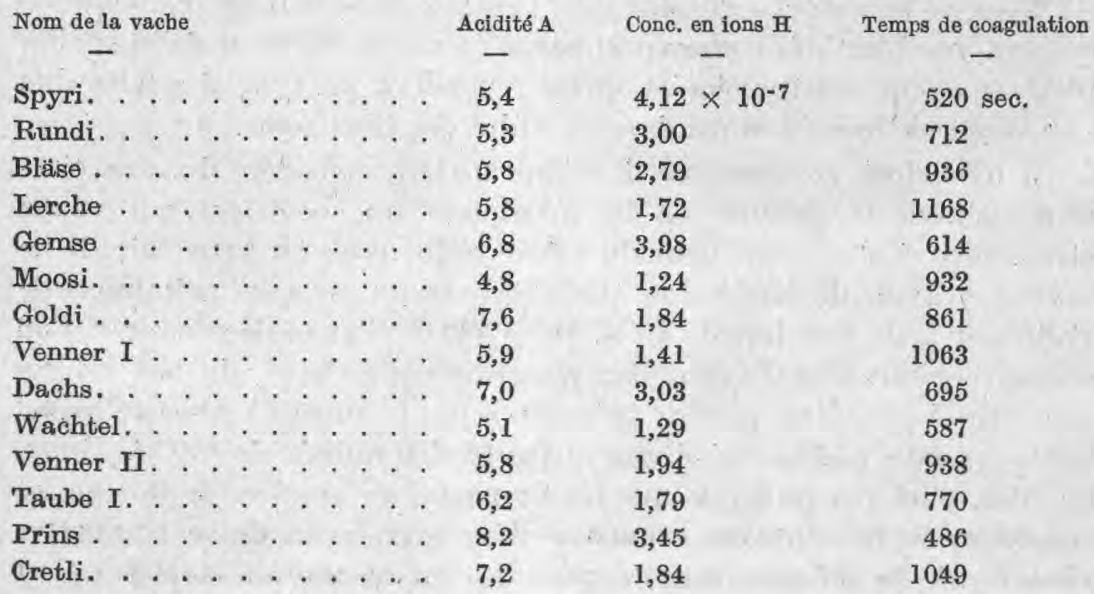

D'un autre côté, pour différents laits d'un même degré d'acidité titrable on obtient, avec la même présure, des temps de coagulation différents. Il faudrait pour expliquer dans le détail les raisons de ces divergences disséquer l'acidité du lait, en distinguer les divers facteurs, et leur attribuer à chacun d'eux la part qui leur revient. Sans aller jusque-là, nous dirons que cette acidité du lait est en gros la somme de deux acidités, celle du caséinate et celle des substances dissoutes dans le sérum. C'est cette dernière qui intervient d'une façon dominante dans le $p \mathrm{H}$ et, par là, dans le phénomène de la coagulation par la présure.

L'INTERVENTION DES IONS H. - VAN DAM (239) estime que la vitesse de la coagulation par la présure est directement proportionnelle à la concentration en ions $\mathrm{H}$. Cette donnée, Allemann s'est d'abord employé à la vérifier, soit en acidifiant le lait par des quantités équivalentes de $\mathrm{HCl}$ ou d'acide acétique. Bien que les degrés d'ionisation de ces acides ne soient pas les mêmes, Allemann constate, lorsqu'on les ajoute à des mêmes quantités de lait en quantités équivalentes, qu'ils

(238) O. Altemans. - Die Bedeutung der Wasserstoffionen für die Milchgerinnung. Biochem. Zeitschr., 1921, 45, 346."

(239) W. VAN DAM. - Beitrag zur Kenntnis der Labgerinnung. Zeitschr. für physiol. Chem., 58, 295. 1908-1909. 
en augmientent l'acidité titrimétrique dans la même proportion, de même qu'ils conduisent à des $p H$ égaux. C'est que le lait est un milieu complexe, possédant un pouvoir tampon, si bien que les ions $H$ actuels comme les potentiels sont liés, et ce qu'on mesure comme ions libres n'est rien autre que les nouveaux ions formés par glissement des phosphates du lait du côté acide en faveur des monophosphates. L'action régulatrice du lait a toutefois une limite dans la transformation du phosphate bimétallique en phosphate monométallique. En effet, l'addition d'ions acides a pour but également de décalcifier le caséinate ce qui se fait presque en même temps que la transformation du phosphate bimétallique en phosphate monométallique, et le moment arrive où il y a une précipitation de caséine, ou mieux, comme nous l'avons vu antérieurement, de caséinate monocalcique, selon la terminologie de L. L. van Slyke et Bosworth.

Pour exclure l'action régulatrice du lait, Allemana utilisa le tampon connu : acétate de sodium + acide acétique, et il en conclut que conformément à l'hypothèse de VAN DAM, les temps de coagulation sont moditiés proportionnellement à la concentration en ions $\mathrm{H}$.

L'intervention des ions Ca. - D'après ses expériences, MunDIGER estime qu'on ne peut pas admettre sans restriction que la vitesse de coagulation soit directement proportionnelle à la concentration en ions $\mathrm{H}$ et qu'un autre facteur doit intervenir : ce sont les ions $C a$.

Ceci ressortira très nettement de recherches sur les complexes que nous exposerons ultérieurement. Nous verrons que des complexes de même $p \mathrm{H}$ coagulent d'autant mieux que leur richesse en ions Ca est plus forte, mais nous saurons en même temps montrer que le rôle des ions $\mathrm{Ca}$ est d'une tout autre nature que celui des ions $\mathbf{H}$.

Les recherches de GrIMMER et Rudzik, d'Allimann, de MUNDIGER, ainsi que les nôtres, montrent combien est différente l'influence d'un degré selon sa place sur l'échelle des acides. Elle est grande lorsqu'on débute; avec telle présure, lorsqu'on passe de l'acidité 7,4 à l'acidité 8,4 (degrés Soxhlet-Henkel), les temps de coagulation tombent rapidement de 5 minutes environ, alors que pour des acidités beaucoup plus marquées, l'influence de $1^{\circ} \mathrm{en}$ plus est à peine sensible.

Mundiger confirme la loi de Griminer relativement à l'influence de l'acidité sur les temps d'emprésurage ; il suit les acidités au $p \mathrm{H}$ : 


\section{TABLEAU XLII.}

VARIATIONS DES TEMPS DE GOAGULATION EN FONGTION DU $p H$ Temps de coagulation

\begin{tabular}{lcc}
$p$ H & Observés & Caleulés \\
\hline 6,9 & 946 & 950 \\
6,8 & 818 & 808 \\
6,7 & 691 & 704 \\
6,6 & 658 & 591 \\
6,5 & 565 & 509 \\
6,4 & 467 & 440 \\
6,3 & 325 & 383 \\
6,1 & 283 & 295 \\
5,9 & 223 & 233 \\
5,8 & 223 & 210 \\
5,7 & 157 & \\
5,6 & 70 &
\end{tabular}

L'emprésurage est donc dépendant : a) de l'acidité, c'est-à-dire du nombre des ions $\mathrm{H}$; b) de la quantité de présure; c) de la nature du lait: d) de la température; enfin: e) de la quantité des ions Ca qu'il renferme.

Influence de la réaction des solutions de présure. - Grimmer et RuDziok ont ajouté un autre facteur : la réaction des solutions de présure. Nous savons que ces auteurs ajoutent à une quantité donnée de $\mathrm{cm}^{3}$ de lait, un nombre de $\mathrm{cm}^{3}$ de la solution de présure parfois considérable, atteignant jusqu’à $50 \%$. Si la présure est nettement acide, on conçoit que les ions $H$ qu'elle apporte dans le mélange puissent influen er considérablement l'emprésurage. Mais est-on en droit de dire que c'est la présure très acide qui agit et ne doit-on pas séparer le ferment de l'acide? La présure très acide étudiée par GrIMmmer et RuDzrK est peut-être d'une nature un peu différente des autres présures commerciales, puisque son fabricant recommande d'ajouter à sa solution un peu de $\mathrm{HCl}$ lors de l'emprésurage du lait dans la fabrication du fromage. Avec cette présure, ou soi-disant présure très acide, la loi de STORCH n'est plus valable : Les produits du temps de la coagulation par la quantité de ferment augmentent plus rapidement au début, puis peu à peu plus lentement. Ils se rapprochent d'une valeur maximum dans une courbe également d'allure logarithmique.

L'influence de la forte acidité de la pseudo-présure étudiée par Grimmer et Rudzick est bien mise en évidence par quelques expériences que nous avons faites avec les macérations chlorhydriques de caillettes préparées au laboratoire. Elles nous montreront une fois de plus la grande sensibilité de l'emprésurage vis-à-vis des acides.

A $25 \mathrm{~cm}^{3}$ de lait, nous ajoutons un $1 / 2 \mathrm{~cm}^{3}$ de la macération de caillettes; la quantité de $\mathrm{HCl}$ apportée par celle-ci correspond à $0 \mathrm{gr} .08$ par litre de lait, 
quantité extrêmement faible, mais qui néanmoins influence nettement le temps de prise, puisque lorsqu'elle disparaît par dialyse, nous voyons celle-ci s'allonger.

TABLEAU XLIII.

Durée de la dialyse de la macération chlorhydrique de présure

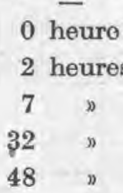

Temps de prise
70 sec.
75
80
100
100

Aucune différence n'est à noter entre les cialyses de 32 et de 48 heures. C'est que l'acide chlorhydrique a entièrement disparu au bout de 32 heures.

Si nous considérons les 16 heures qui s'écoulent entre les deux derniers essais, nous voyons qu'ils ont donné des temps de prise identiques ; nous pouvons conclure que la présure proprement dite n'a nullement perdu de sa force et qu'elle n'a pas traversé la membrane du dialyseur. Peut-être qu'avec des dialyses plus prolongées, en ayant recours à des dialyseurs à parois très perméables, obtiendrait-on des diminutions dans la force de la présure, ce que nous traduirions en disant qu'une certaine quantité de celle-ci a pu, avec le temps, traverser la membrane.

Il y a lieu de comparer l'acide chlorhydrique et l'acide acétique, c'est-à-dire un acide minéral et un acide organique, à l'acide lactique qui est l'acide régulier des acidifications spontanées du lait. Les quantités des acides qu'on ajoute au lait originel nous permettent d'augmenter cette acidité, degré par degré Dornic ; nous pourrons suivreaussi cette aciditéavec le potentiomètre. On peut même ajouter l'acide d'une façon très mesurée pour que l'acidité ne s'accroisse que par quart de degré Dornic (a).

L'acidité, la consistance du caillé et la synérèse. - Au fur et à mesure que l'acidité s'élève, la consistance du caillé devient plus terme en même temps que la synérèse s'accuse plus rapidement.

Voiciles diverses expériences que nous avons faites sur cette question : l'acidité du lait originel était de $21^{\circ} \mathrm{D}$.

\section{TABLEAU XLIV}

VARIATIONS DU TEMPS DE PRISE EN FONCTION DE L'AGIDITÉ (PRÉSURE FORTE)

$\begin{array}{cccc}\text { Degré D } & \text { Temps de prise } & \text { Degré D } & \text { Temps de prise } \\ 2^{\circ} & 290 \text { sec. } & 29^{\circ} & 60 \text { see. } \\ 22^{\circ} & 250 & 30^{\circ} & 50 \\ 23^{\circ} & 200 & 31^{\circ} & 45 \\ 24^{\circ} & 160 & 32^{\circ} & 40 \\ 25^{\circ} & 125 & 33^{\circ} & 35 \\ 26^{\circ} & 100 & 34^{\circ} & 30 \\ 27^{\circ} & 80 & 35^{\circ} & 25 \\ 28^{\circ} & 70 & 36^{\circ} & 25\end{array}$

(a) Dans nos promières recherches, vieilles déjà de près desix ans. nous déterminions l'acidité titrimétriquement. Puis, nous avons utilisé le potentiomètre. On trouvera ici des résultats exprimés de l'une et del'autre manière, soit isolés, soit accolés. 
Le graphique $\mathrm{V}$ traduit les résultats du tableau XLIV.

GRAPHIQUE V.

INFLUENCE DE L'AGIDITÉ LAGTIQUE SUR L'EMPRÉSURAGE (PRÉSURE FORTE)

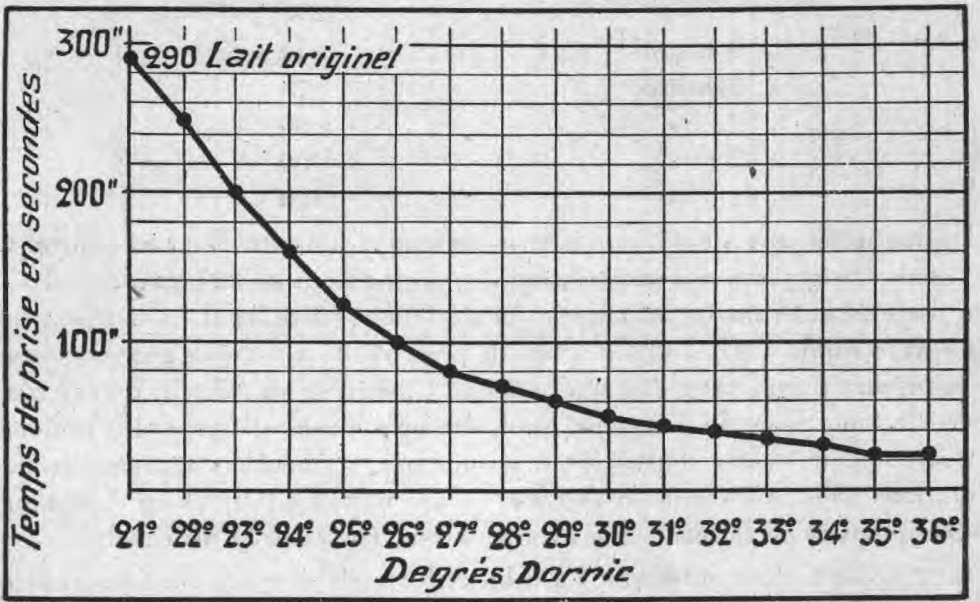

Nous avons opéré avec le même lait, mais en prenant une présure trois fois plus faible pour augmenter les temps de coagulation.

Voici quels sont les temps de coagulation :

TABLEAU XLV

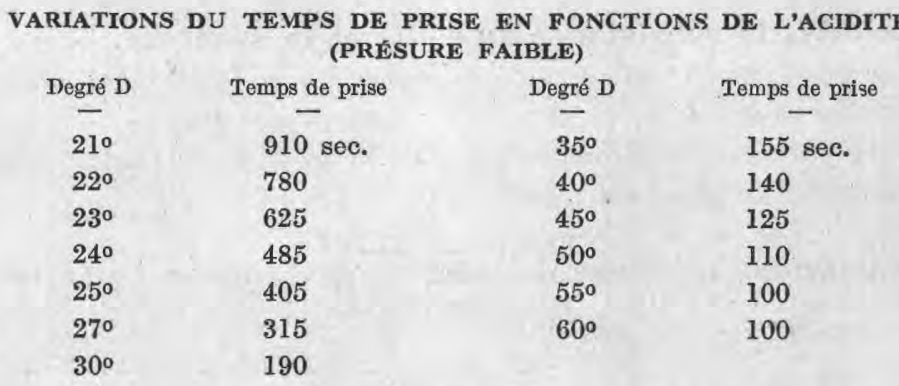

Avec 55० D., commence la précipitation par les acides seuls; en effet, le lait porté au B.M. avant toute addition de présure, précipitait partiellement.

La courbe VI reproduit graphiquement les données du tableau qui précède. La comparaison avec la précédente n'est pas dépourvue d'intérêt. En effet, lorsque la présure est forte, la courbe a une allure asymptotique, est plus couchée sur la ligne des abscisses, se relève moins vers celle des ordonnées ; mais quand la présure est faible et que le temps de prise initial est grand, on saisit bien mieux l'influence de l'acidité. 


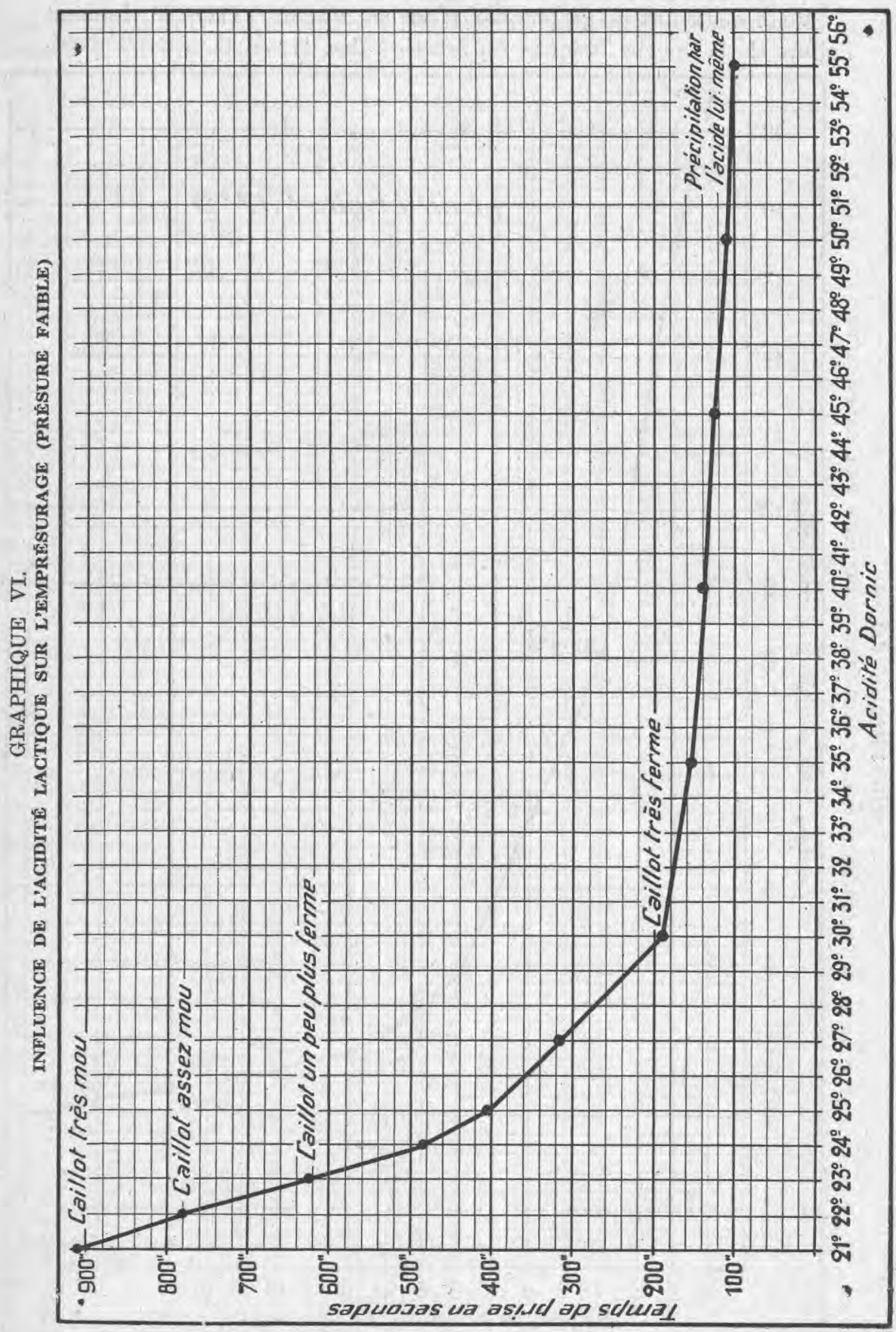


Voici deux autres graphiques (Courbes VII et VIII) qui donnent l'allure de la courbe obtenue en fonction des degrés D. ajoutés expé-

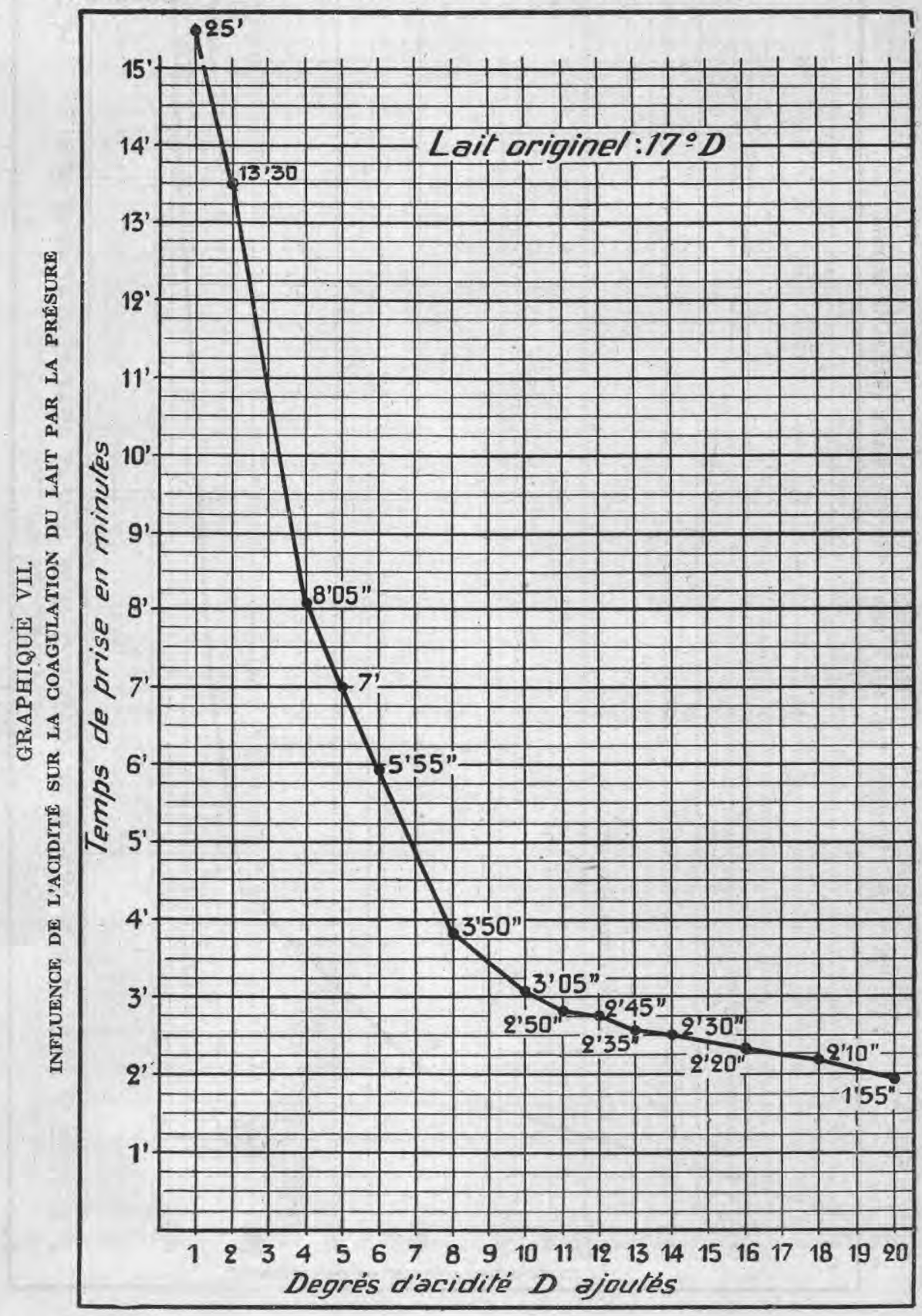




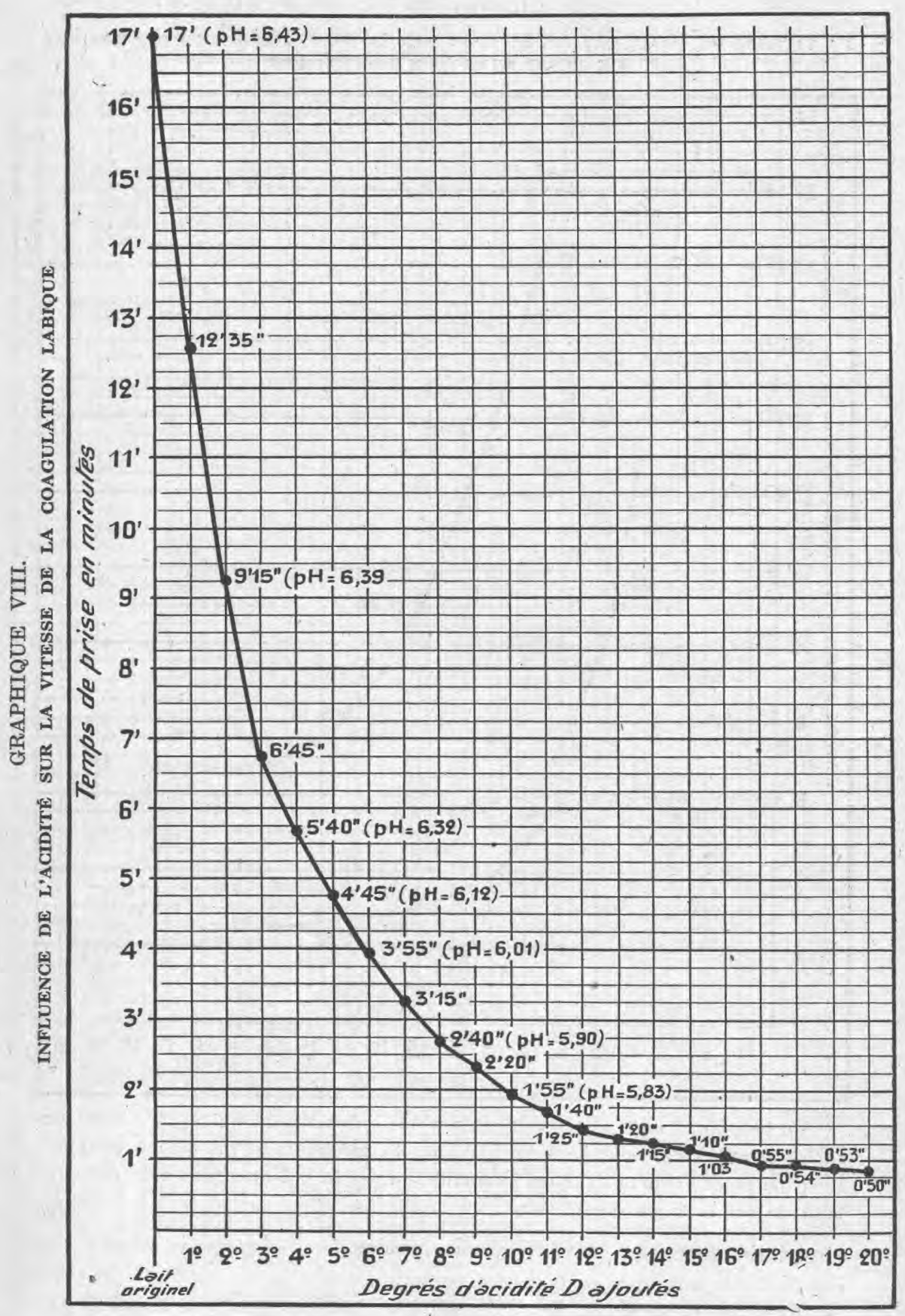


GRAPHIQUE IX.

LA VITESSE DE COAGULATION DU LAIT PAR LA MÊME PRÉSURE A DES DEGRES DE CONGENTRATION DIFFERENTS

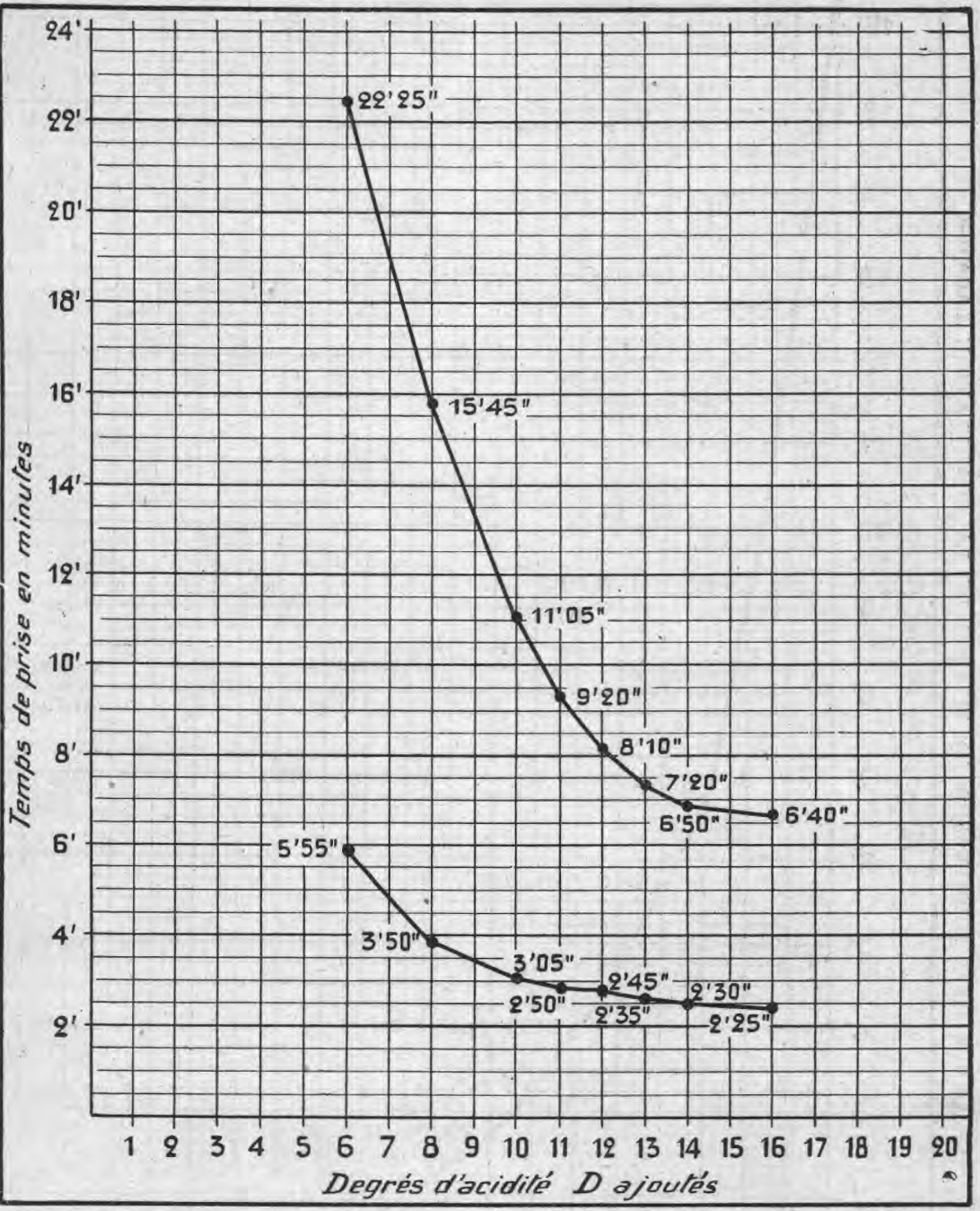

Ce graphique nous permet de renouveler l'observation qui vient d'être formulée à propos des courbes $V$ et VI. Avec la présure faible, la courbe se relève plus nettement vers la ligne des ordonnées; avec la présure forte, elle est plus couchée sur la ligne des abcisses.

터영 rimentalement. Les $p H$ de certains points de la deuxième courbe ont été obtenus par la voie potentiométrique.

Lorsque l'accroissement de l'acidité se fait par guart de degré $D$, et 
GRAPHIQUE $X$.

ACCROISSEMENT DE L'ACIDITÉ PAR 1/4

DE DEGRÉ D.

qu'on emploie des présures faibles, avec lesquelles il est plus facile comme nous venons de le voir, de saisir l'influence de l'acidité, on se rend aisément compte une fois de plus de la grande importance de celle-ci.

Comparaison de divers acides : acétique, chlorhydrique et lactique. - Dans la comparaison des actions de l'acide acétique, de l'acide chlorhy.

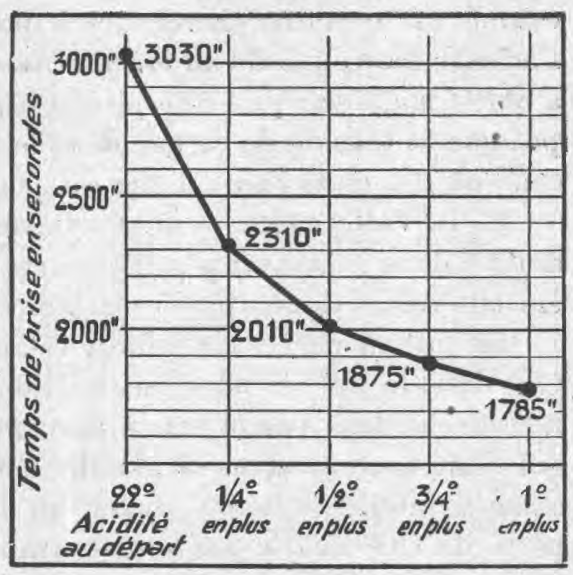

GRAPHIQUE XI.

COMPARAISON DES ACIDES LAGTIQUE, CHLORHYDRIQUE ET AGÉTIQUE

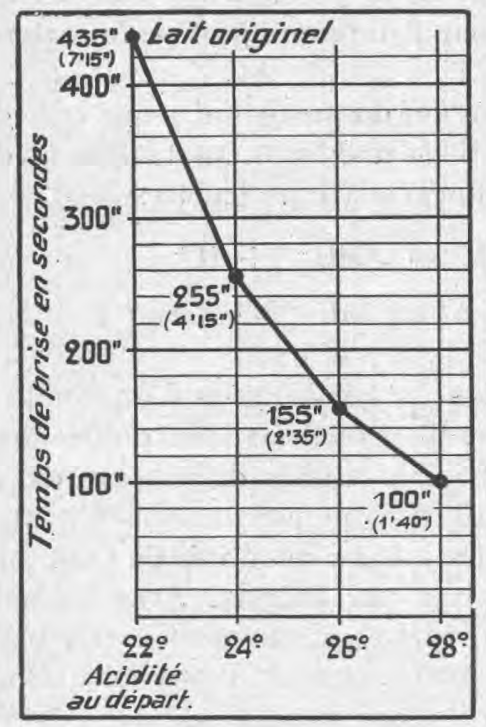

drique et de l'acide lactique, on peut a priori penser qu'en raison de la dilution faible de ces acides dans le lait, leur ionisation doit être identique et par suite identiques les résultats; c'est ce que l'expérience a montré.

Des quantités équimoléculaires de ces trois acides ont été utilisées pour acidifier le lait, de deux degrés en deux degrés $D$.; aucune différence n'est perçue.

\section{ACTION DE L'AGIDE GARBONIQUE SUR L'EMPRÉSURAGE}

L'ACIDE CARBONIQUE DU LAIT. - Plusieurs auteurs avaient déjà remarqué que, dans les heures qui suivent la traite, le lait se modifie un peu dans sa façon de se comporter vis-à-vis de la présure. On doit 
d'abord penser qu'en prenant contact avec l'air, il a perdu une partie de ses gaz. Parmi eux, il faut citer en premier lieu l'anhydride carbonique dont le taux en volume par litre de lait est assez élevé, puisqu'il est estimé par certains chercheurs à près de $200 \mathrm{~cm}^{3}$. Ce dernier chiffre s'adresse bien entendu au lait pris dans la mamelle, car dès que ce liquide a quitté la glande qui l'a sécrété, il perd la plus grande partie de son $\mathrm{CO}^{2}$ puisque la tension de ce gaz dans le lait est beaucoup plus grande que celle qu'il a dans l'air où elle est pour ainsi dire négligeable.

L. L. VAN SLYKE et J. C. BAKER (240) estiment que la tension de $\mathrm{CO}^{2}$ dans le lait est à peu près égale à 50-55 millimètres de mercure. Le lait serait done approximativement une solution $\mathrm{N} / 100$ de $\mathrm{CO}^{3} \mathrm{H}^{2}$.

Le travail de ces auteurs a éclairé les esprits sur l'importance de $\mathrm{CO}^{2}$ dans le lait. Il nous laisse évidemment penser que le liquide que nous recueillons par la traite ne peut être strictement identifié à celui qui était contenu dans la glande, à l'intérieur des acinis et des petits canaux intraglobulaires, avant qu'il ait pris contact avec l'air. 'La perte de $\mathrm{CO}^{2}$ ne va pas sans entraîner des modifications dans l'équilibre salin du lait tel qu'il a été sécrété. Il y aurait done intérêt à entreprendre une étude complète de ce côté; nous en marquons simplement ici la nécessité.

Quoi qu'ilen soit, l'importance que nous y attachons nous a engagé à voir quels résultats donnerait pour l'emprésurage l'acidification du lait par $\mathrm{CO}^{2}$.

L'action de $\mathrm{CO}^{2}$ sur le lait est a priori du même ordre que celle d'un acide non volatil soluble dans l'eau. En se dissolvant partiellement dans l'eau du lait, $\mathrm{CO}^{2}$ crée des ions $\mathrm{H}$ selon l'équation connue :

$$
\mathrm{CO}^{2}+\mathrm{H}^{2} \mathrm{O}=\mathrm{CO}^{3} \mathrm{H}^{2}=\mathrm{CO}^{3} \mathrm{H}^{-}+\mathrm{H}^{+}
$$

En même temps, il modifie l'équilibre salin préexistant en solubilisant une partie des sels de calcium.

Nous avons fait barboter $\mathrm{CO}^{2}$ dans le lait à raison d'une bulle par seconde environ pour $100 \mathrm{~cm}^{3}$ de lait. Il est facile avec un double détendeur sur la bombe d'obtenir cette faible vitesse du passage du gaz. Evidemment, avec $\mathrm{CO}^{2}$ qui est volatil, nous ne pouvons pas être assuré de toujours dissoudre dans le lait les mêmes quantités de $\mathrm{CO}^{2}$, toutes conditions nous paraissant semblables par ailleurs. Avec les acides lactique, acétique et chlorhydrique, nous sommes tout à fait maître de notre acidification. Nous ne le sommes que fort peu lorsque nous employons $\mathrm{CO}^{2}$; néanmoins, il est encore possible de procéder par comparaison.

Le lait originel a $19^{\circ}$ D. ; des prises de lait ont été faites successivement après

(240) L. L. SLYKR et J. C, BAKER. - Carbonic acid and carbonates in cow's milk, New-York Agric. Exp. Stat. Juin 1919., Techn. Bull, n 69. 
barbotage de 1 minute, 2 minutes, 5 minutes, 10 minutes et 30 minutes ; en même temps, on prenait l'acidité correspondante et on emprésurait.

Il en est ici comme de tous les cas où l'acidité est augmentée par l'apport d'un acide fixe. Le caillé est d'autant plus ferme que la prise est plus rapide.

Voici les chiffres obtenus :

\section{TABLEAU XLVI}

\section{AGTION FAVORABLE DE CO $^{2}$ SUR L'EMPRÉSURAGE}

Acidité $\mathrm{D}$, Temps de prise

Leit originel. . . . . . . . $19 \quad 210 \mathrm{sec}$

Après passage de $\mathrm{CO}^{2}$.......

Passage pendant : $1 \mathrm{~min}$...... $22 \quad 155$

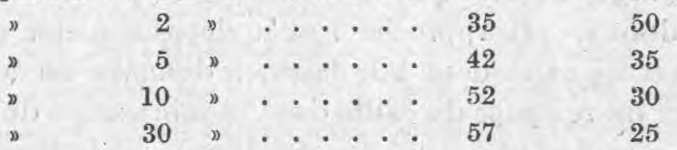

Le graphique joint donne les résultats fort intéressants obtenus.

GRAPHIQUE XII.

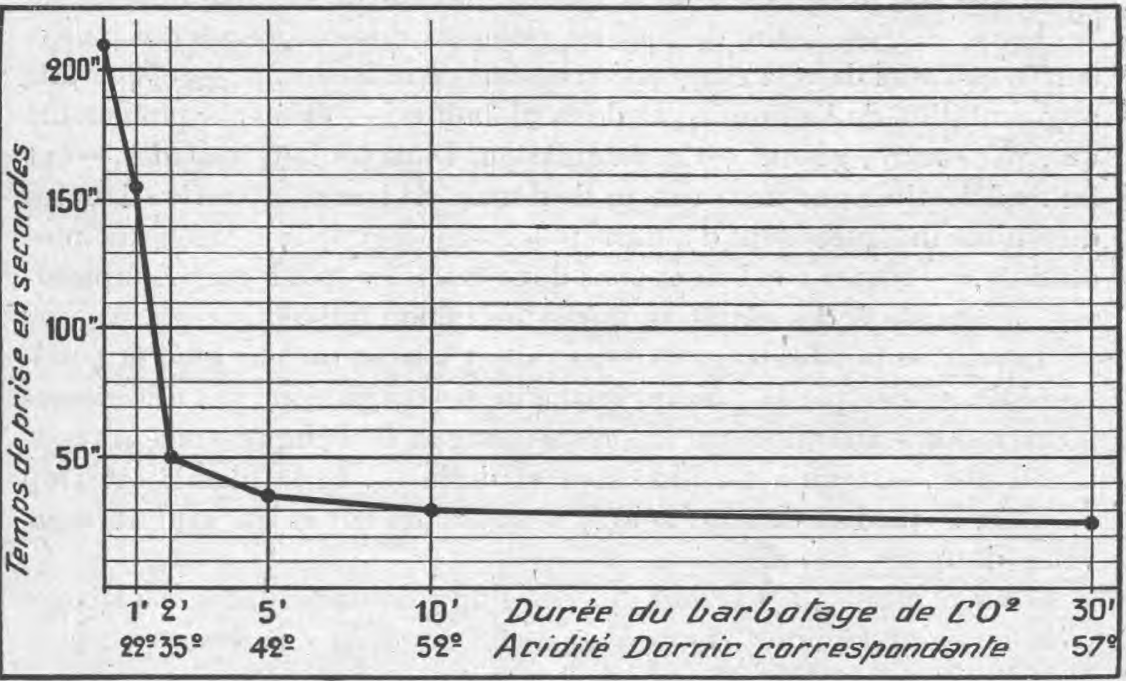

Le rôle de $\mathrm{CO}^{2}$ est double. - Nous voyons qu'un seul barbotage de deux minutes à la faible vitesse que nous avons indiquée ci-dessus : une bulle par seconde pour $100 \mathrm{~cm}^{3}$ de lait, améliore considérablement l'emprésurage, puisqu'il réduit le temps de prise de plus des troisquarts, de 210 secondes à 50 secondes. Le rôle du $\mathrm{CO}^{2}$, comme d'ailleurs celui des autres acides dont il a été parlé auparavant, est toujours double. 
a) D'une part, avons-nous vu, il crée des ions $H$, et engendre ainsi une acidité réelle;

b) D'autre part, à la faveur de cette acidité, il contribue à solubiliser les sels de chaux du lait, à transformer les sels insolubles tels que le phosphate tricalcique en sels solubles : phosphate bi- et surtout phosphate monocalcique, toutes circonstances qui ne peuvent que faciliter l'emprésurage.

Nous reviendrons sur ce point iorsque nous étudierons la mécanique chimique des phosphates de calcium, car nous verrons ce que deviennent ces phosphates sous l'influence du barbotage carbonique.

Le barbotage earbonique dans les laits paresseux. - Les laits dits alcalins dont le $p \mathrm{H}$ approche 7 et le dépasse même coagulent fort mal par la présure et tardent à le faire en donnant un coagulum mou, si bien que lors du rompage du caillé dans la fabrication du fromage, on a des pertes considérables; et encore, nous ne tenons pas compte ici de la diminution de qualité du produit fabriqué. Ces laits sont dits "paresseux"). Ils proviennent le plus généralement, pour ne pas dire toujours, de mamelles malades. Au point de vue chimique, ils se caractérisent par une diminution de la caséine, de l'acide phosphorique et de la chaux, — c'est-à-dire de tous les éléments dits coagulants qui jouent le rôle essentiel dans la coagulation du lait en la facilitant, - et par une augmentation de l'albumine et de la globuline, - éléments protecteurs qui, par contre, gênent cette coagulation. Dans les laits malades, - et les modifications que nous venons de donner de leur composition dans les directions indiquées sont d'autant plus marquées que la mamelle est plus atteinte, - toutes conditions sont done réalisées pour que l'emprésurage aboutisse à des résultats médiocres, sinon nuls.

Lorsqu'on fait barboter $\mathrm{CO}^{2}$ dans de tels laits, on leur rend la possibilité de gélifier par la présure lorsqu'ils ne coagulaient pas antérieurement, ou on raffermit le caillé lorsque, coagulant, l'emprésurage n'a boutissait chez eux qu'à un bloc mou et diffluent. Si la glande est trop touchée, le produit de sécrétion(?) n'est plus du lait et ilne saurait intervenir dans ces considérations.

Nous pensons que $\mathrm{CO}^{2}$ aura sa place un jour danz l'industrie laitière pour hâter et faciliter l'emprésurage de ces laits "paresseux ": laits malades, laits alcalins, Il y a plusieurs années que nous avons appelé l'attention sur cette question, mais nous ne croyons pas que la pratique s'en soit préoccupée. Un jour viendra sans doute où la bombe à gaz carbonique fera partie du matériel des fromageries.

En fait, les deux résultats auxquels conduit l'emploi de $\mathrm{CO}^{2}$, c'està-dire l'augmentation d'acidité du milieu et celle des sels ealciques solubles dans le lait, sont intimement liés et ne peuvent être séparés l'un de l'autre. 


\section{ACTION DE LA PRÉSURE SUR DES LAITS ALCALINISÉS.}

Si l'acidité favorise, et considérablement peut-on dire, l'action de la présure, inversement, l'alcalinité ne peut que nuire à l'emprésurage. amollir le caillé, retarder et même supprimer la coagulation.

L'alcalinisation du lait peut s'effectuer au moyen de bases alcalines : potasse, soude ou ammoniaque; de bases alcalino-terreuses : chaux, baryte ou strontiane, ou encore de magnésie.

A priori, sachant l'heureuse influence des sels calciques sur la coagulation, on peut penser que l'alcalinisation par la chaux retentira moins sur l'emprésurage que l'alcalinisation par les bases alcalines, fixes ou volatiles.

Nos expériences ont été effectuées avec la soude, l'ammoniaque et la chaux selon le protocole suivant : Le lait originel, écrémé toujours a été additionné de base : soude, ammoniaque ou chaux, de façon que son acidité soit abaissée degré par degré D. Encore une fois, il est tenu compte de la dilution du lait.

ALCALINISATION PAR LA soude. - Lorsqu'il s'agit de soude ou d'ammoniaque, la dilution qu'entraine l'addition de la base au lait est pour ainsi dire négligeable, car on peut utiliser ces alcalis fortement concentrés Pour la chaux, il n'en est pas de même.

Voici les résultats obtenus, d'abord avec la soude.

TABLEAU - XLVII.

AGTION DE LA SOUdE SUR LA COAGULATION DU LAIT PAR LA PRÉSURE

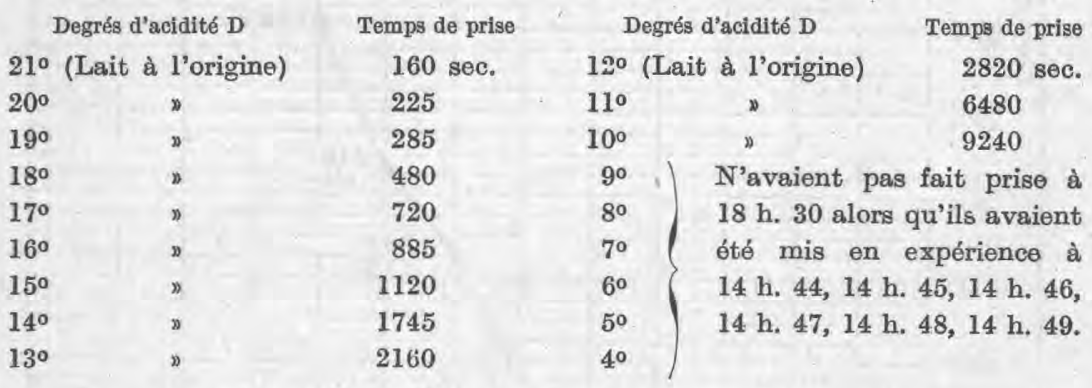

On se rend compte par cette expérience si nette de l'influence très retardatrice de l'alcalinisation du lait réalisée par un alcali fixe.

Une simple baisse d'acidité de 1 degré allonge nettement le temps de prise, mais cet allongement est surtout notable pour les alcalinisations très prononcées. C'est ainsi qu'en passant de $12^{\circ}$ à $11^{\circ}$, le temps de prise a plus que doublé. Ceci répond d'ailleurs tout à fait à ce que l'on sait de l'influence favorable de l'acidité sur la coagulation par la présure ; ce sont les premières acidifications qui marquent le plus nettement leur action sur le temps de prise. 
TEMPS DE PRISE PAR LA PRÉSURE D'UN LAIT ADDITIONNÉ DE SOUDE

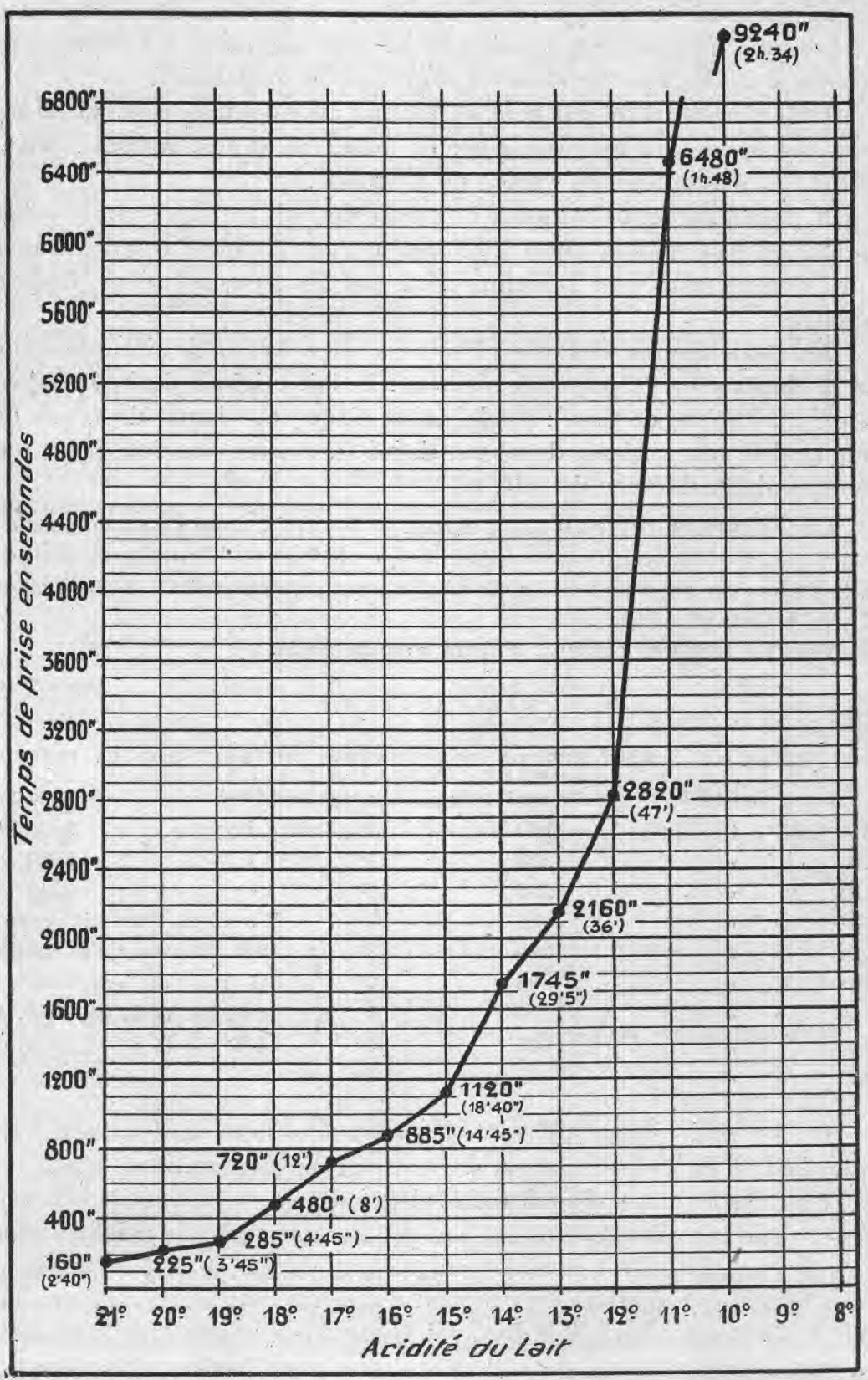


Dans cette expérience, nous avons comparé, en somme, des échantillons d'un même lait alcalinisé à des titres divers.

$\mathrm{Si}$ nous comparons un lait alcalinisé à un autre lait également alcalinisé, rien ne dit que nous obtiendrons des résultats identiques pour des mêmes degrés titrimétriques. Les laits ne se ressemblent pas tous à l'état normal, et nous savons que pour une même réaction titrimétrique, c'est-à-dire pour une même acidité potentielle, ils peuvent avoir des $p H$ différents, c'est-à-dire des acidités actuelles non identiques, quoique voisines. Dans ces conditions, ils ne réagissent pas de façon semblable dans les mêmes circonstances, après addition de la même quantité de la même présure. Les laits dits alcalins, d'une façon générale coagulent plus tardivement que les autres, mais des laits alcalins de diverses origines ayant titrimétriquement le même degré $\mathrm{D}$, peuvent très bien coaguler avec la même présure au bout de temps différents.

ALCALINISATION PAR L'AMMONIAQUE. - Nous avons utilisé l'ammoniaque dans les mêmes conditions que la soude, c'est-à-dire que nous avons abaissé l'acidité originelle du lait degré par degré.

\section{TABLEAU XLVIII.}

\section{AGTION DE L'AMMONIAQUE SUR L'EMPRÉSURAGE}

\begin{tabular}{|c|c|c|c|c|c|c|}
\hline \multicolumn{2}{|r|}{ Acidité } & \multirow{2}{*}{$\begin{array}{l}\text { Temps de prise } \\
95 \text { sec. }\end{array}$} & \multicolumn{3}{|c|}{ Acidité } & \multirow{2}{*}{$\begin{array}{r}\text { Temps de prise } \\
1170 \mathrm{sec}\end{array}$} \\
\hline $20^{\circ}$ & (Lait originel) & & $13^{\circ}$ & (Lait & originel) & \\
\hline $19^{\circ}$ & $\infty$ & 120 & $12^{\circ}$ & & " & 1620 \\
\hline $18^{\circ}$ & D & 155 & $11^{\circ}$ & & ” & 2100 \\
\hline $17^{\circ}$ & n & 200 & $10^{\circ}$ & & $n$ & 2700 \\
\hline $16^{\circ}$ & ” & 250 & $9^{\circ}$ & & ” & 3420 \\
\hline $15^{\circ}$ & v & 465 & $8^{\circ}$ & & y & 4200 \\
\hline $14^{\circ}$ & 0 & 780 & 70 & & » & 5100 \\
\hline
\end{tabular}

Après, il n'y a plus de coagulation. Les résultats ont done la même allure qu'avec la soude.

ACTION DE LA CHAUX. - Si l'on utilise l'eau de chaux pour abaisser l'acidité du lait, degré par degré $\mathrm{D}$, on arrive pour les abaissements notables à des dilutions assez fortes.

Dans nos premières expériences faites avec de l'eau de chaux, nous avons obtenu les résultats suivants :

\section{TABLEAU XLIX.}

AGTION DE LA GHAUX SUR LA GOAGULATION LABIQUE

Acidité D

$\begin{array}{cc}20^{\circ} & \text { (lait originel) } \\ 19^{\circ} & n \\ 18^{\circ} & \\ 17^{\circ} & \end{array}$

Temps de prise Aspect des caillés

Caillés

assez

fermes 
GRAPHIQUE XIV.

TEMPS DE PRISE PAR LA PRÉSURE D'UN LAIT ADDITIONNÉ D'AMMONIAQUe

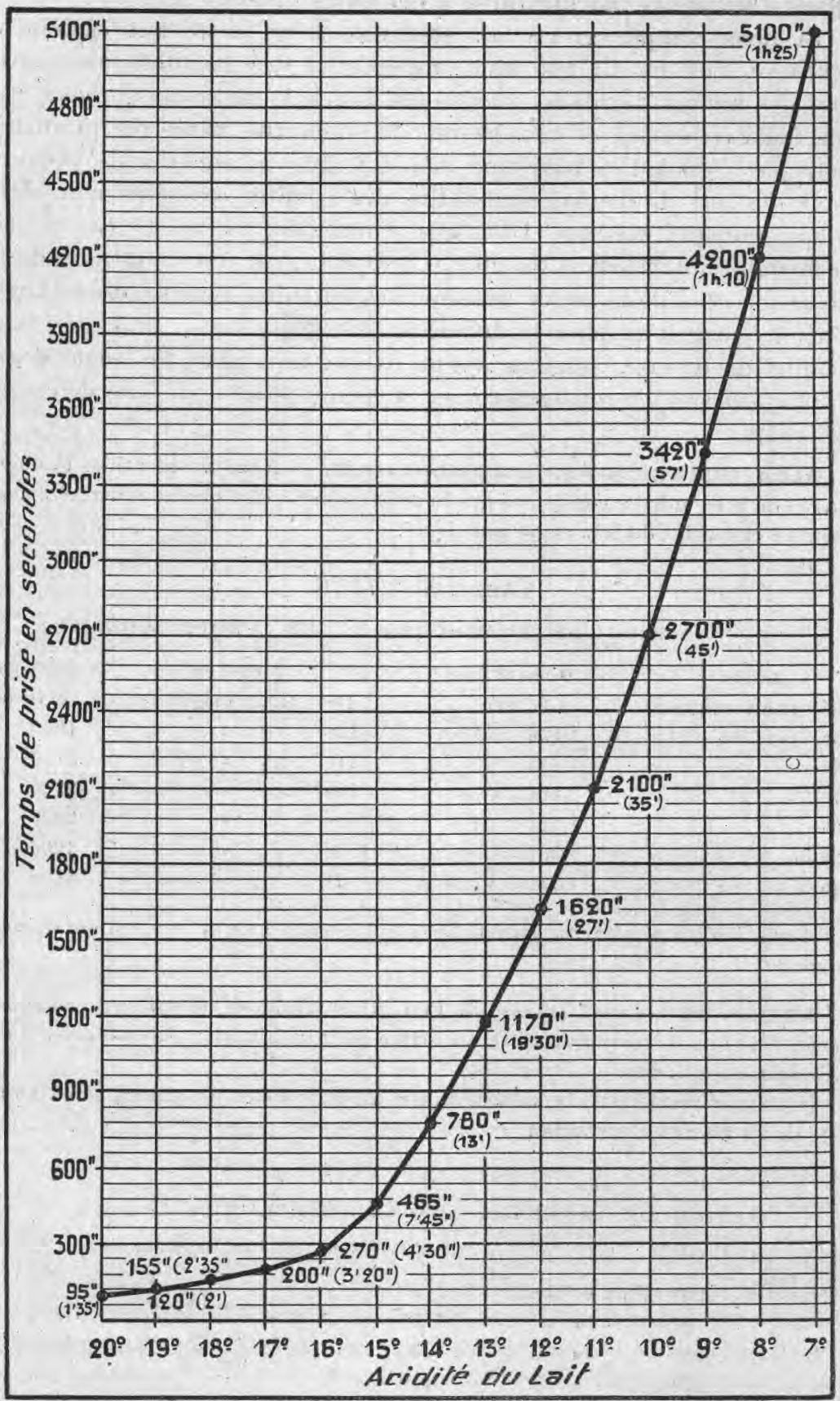


GRAPHIQUE $X V$.

TEMPS DE PRISE PAR LA PRESURE D'UN LAIT ALGALINISÉ PAR L'EAU DE GHAUX

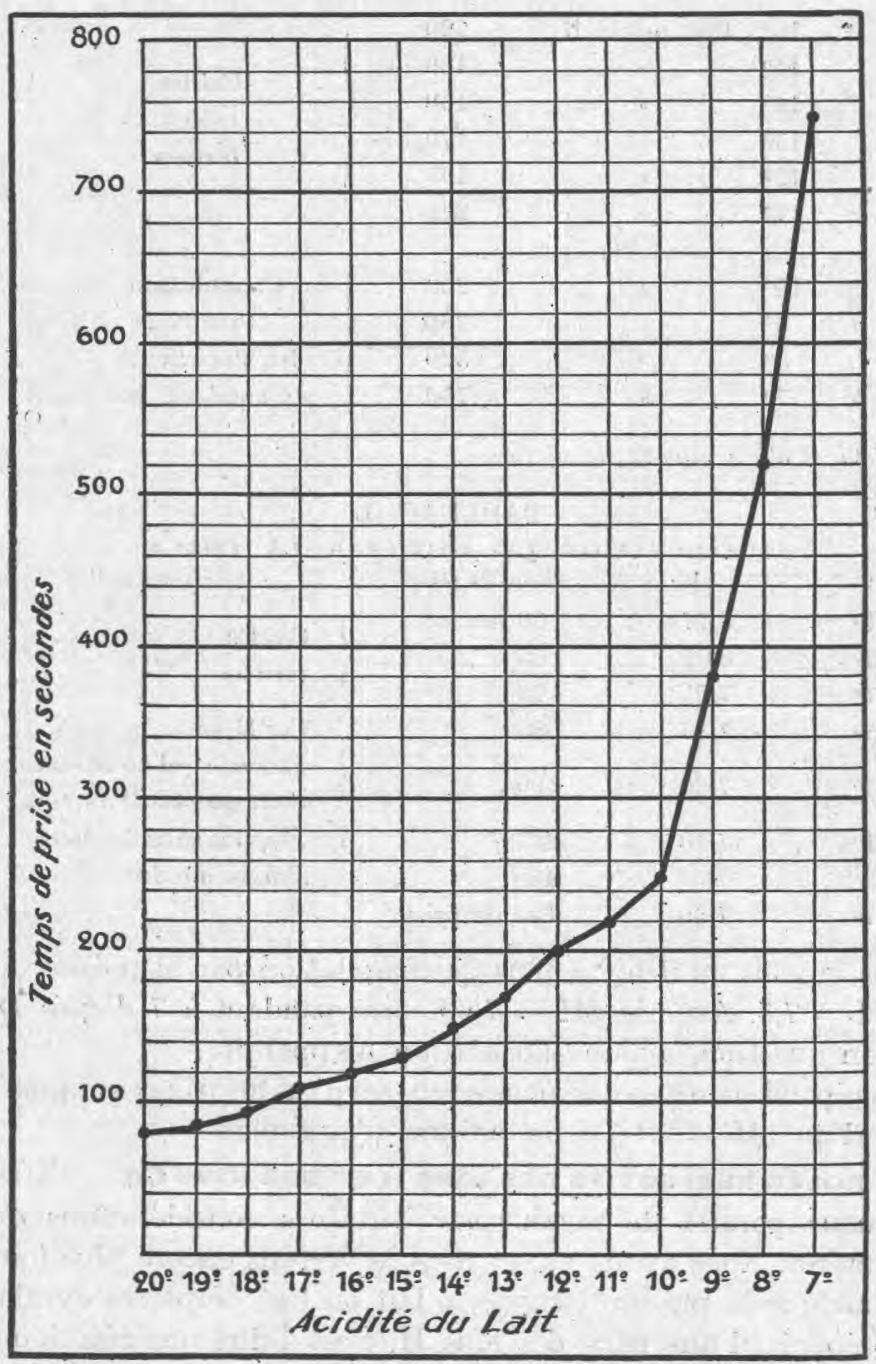

Au lieu de prendre de l'eau de chaux, et afin de supprimerl'inconvénient d'une grande dilution inévitable, nous avons ajouté au lait de la chaux pure calcinée extemporanément. La quantité de chaux nécessaire est placée dans un mortier et broyée soigneusement dans 1 ou $2 \mathrm{~cm}^{3}$ de lait, lequel, sous l'action de cet apport alcalin important, devient translucide et prend rapidement l'aspect de colle. Quand la chaux est dissoute dans les premiers $\mathrm{cm}^{3}$ de lait utilisé, on apporte peu à peu les autres, tout en continuant à broyer et ainsi on répartit la chaux uniformément dans tous les échantillons mis en expérience. 
TABLEAU XLIX (suite).

\begin{tabular}{|c|c|c|c|}
\hline Acidité I & & Temps de prise & Aspect des cai \\
\hline $16^{\circ}$ & (lait originel) & 120 & \\
\hline $15^{\circ}$ & " & 130 & \\
\hline $14^{\circ}$ & " & 150 & Caillés \\
\hline $13^{\circ}$ & 》 & 170 & moins \\
\hline $12^{\circ}$ & n & 195 & fermes \\
\hline $11^{\circ}$ & " & $\begin{array}{l}220 \\
\end{array}$ & \\
\hline $10^{\circ}$ & $n$ & 250 & Coagulation \\
\hline $9^{\circ}$ & 》 & 380 & tardive ; \\
\hline $8^{\circ}$ & 》 & 520 & les flocons ne \\
\hline $7^{0}$ & " & 750 & se soudent pas \\
\hline
\end{tabular}

Au delà, il n'y a plus de floculation.

\begin{tabular}{|c|c|c|c|c|}
\hline & ALGALINISATION & DU LAIT & PAR & GHAUX \\
\hline Acidité D & $p$ H & Temps de prise & & Aspect des caillés \\
\hline $21^{\circ}$ & 6,77 & 50 sec. & ) & \\
\hline $19^{\circ}$ & 6,91 & 65 & & Lantes \\
\hline $17^{\circ}$ & 7,05 & 75 & ) & termes \\
\hline $15^{\circ}$ & 7,19 & 85 & . & Caillé mou \\
\hline $13^{\circ}$ & 7,30 & 100 & \} & $\begin{array}{l}\text { Flocons qui ne se sou- } \\
\text { dent que tardivement }\end{array}$ \\
\hline $11^{\circ}$ & 7,40 & 225 & ) & Flocons sans tendance \\
\hline $9^{\circ}$ & 7,52 & 450 & j & à la soudure \\
\hline 70 & 7,60 & Pas de prise. & & $2=$ \\
\hline
\end{tabular}

Nous voyons qu'il n'y a plus de coagulation par la présure au delà d'un $p \mathrm{H}=7,5$. Avec le $p \mathrm{H}=7,60$ correspondant à 7 degrés $\mathrm{D}$. pour le lait en question, aucune floculation n'apparaît.

Cela répond bien à ce que nous a vons vu dans les pages qui précèdent : au-delà d'un $p \mathrm{H}=7,4-7,5$, la présure n'agit plus.

LES ROLES RESPECTIFS DES IONS H ET DES IONS Ca. - Cette expérience nous permet de revenir sur certaines considérations du plus grand intérêt. Nous avons vu, et nous le verrons encore plus loin, qu'il faut, pour que la présure coagule le lait ou les complexes synthétiquement préparés, d'une part, des ions $H$, c'est-à-dire une réaction convenable et, d'autre part, des ions Ca. Nous verrons encore à dissocier l'action propre de chacun de ces ions, mais retenons d'ores et déjà qu'en présence d'une masse plus que suffisante d'ions $\mathrm{Ca}$, dès l'instant où la réaction de la liqueur dépasse un certain $p \mathrm{H}: 7,4-7,5-7,5$ parait être l'extrême limite, - il n'y a pas de lyse de la caséine sous l'influence du ferment-lab. D'un autre côté, si nous avons un $p H$ convenable, mais insuffisamment d'ions $\mathrm{Ca}$, nous réaliserons bien le clivage de la protéine, mais nous ne provoquerons pas la précipitation du paracaséinate calcique. 
GRAPHIQUE XVI.

TEMPS DE PRISE PAR LA PRÉsure d'UN LAIT ALGalinisé PAR LA GHAUX

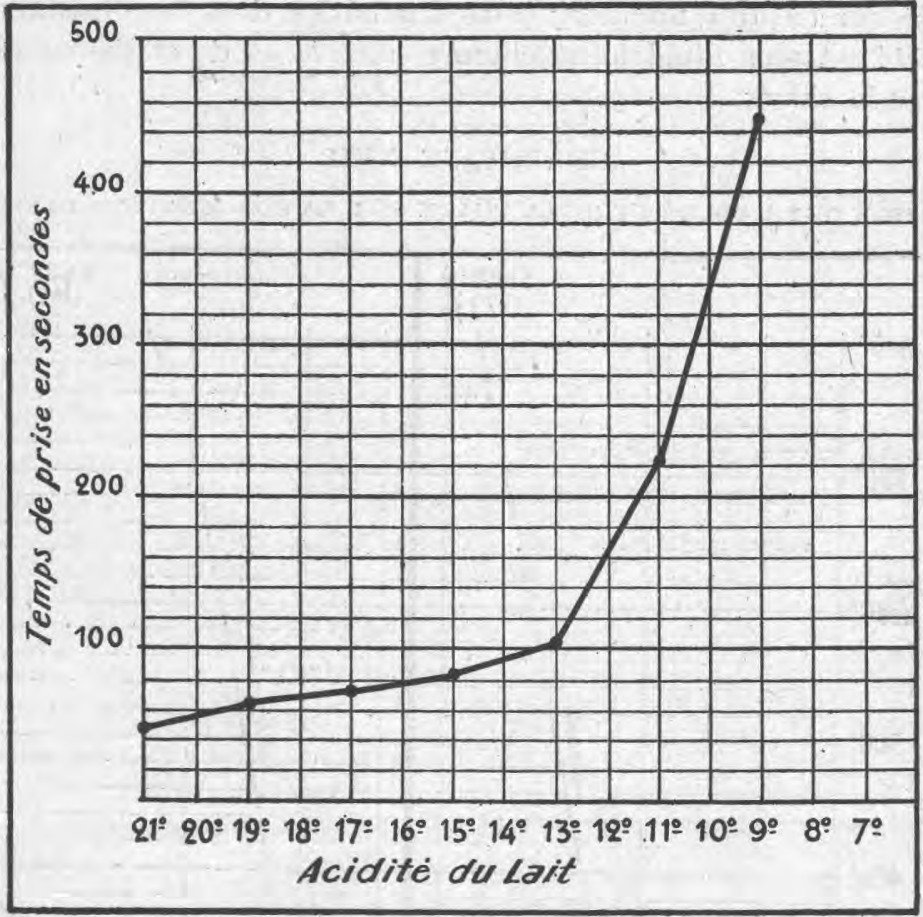

Les ions $H$ sont indispensables au premier temps, - le temps essentiel, redisons-le encore, - de la coagulation du lait, c'est à-dire-le dédoublement de la caséine en paracaséine et protéose soluble. Les ions Ca sont réclamés uniquement par le second temps: la précipitation du paracaséinate.

Ainsi que nous l'avons fait remarquer tout à l'heure a priori, nous pouvons constater, à réactions égales, une différence notable entre l'action de la chaux et celle des bases fixes ou volatiles.

TABLEAU LI

GOMPARAISON DE LA GHAUX ET DE LA SOUDE DANS LEUR ACTION DÉFAVORABLE SUR L'EMPRÉSURAGE

Temps de prise:

Lait originel. . . . . . . . . . . . .

$1^{\text {re }}$ expérience

$2^{\text {me }}$ expérience

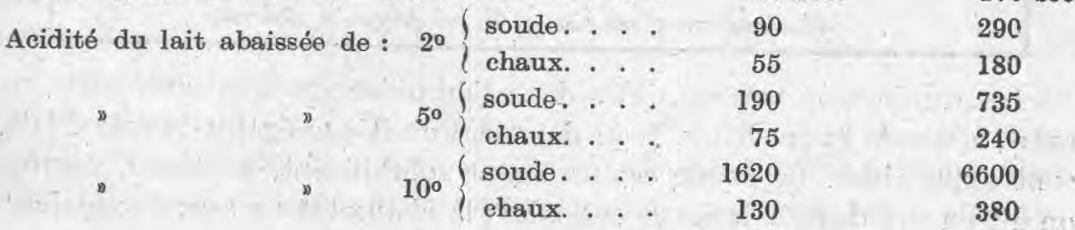


Pour toute alcalinisation, qu'elle soit effectuée par la soude, l'ammoniaque ou la chaux, le caillé est de moins en moins ferme, au fur et à mesure que l'acidité diminue; cette diminution dans la consistance du gel lacté est plus rapidement obtenue avec la soude et l'ammoniaque qu'avee la chaux.

\section{GRAPHIQUE XVII.}

AGTION DE LA SOUDE ET DE LA GHAUX SUR LA GOAGULATION LABIQUE

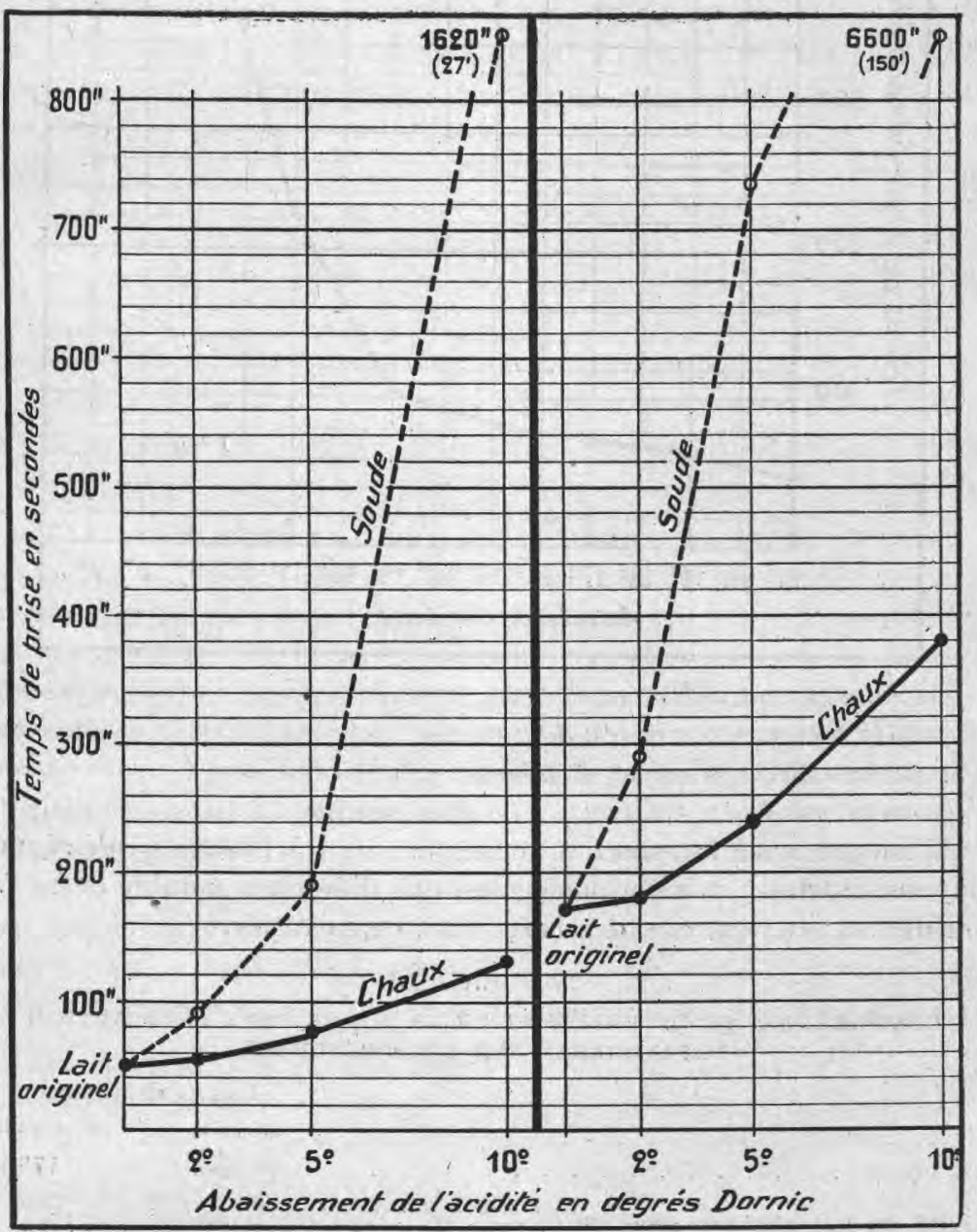

Les différences très sensibles dans l'emprésurage que nous relevons entre la soude et la chaux, pour des alcalinisations égales du même lait, sont explicables. La soude est un agent solubilisant, peptisant, comme on dit encore dans le langage colloïdal; la chaux est un agent coagulant. 
Les sels de sodium de la paracaséine qui se forme sous l'action de la présure sont solubles; les sels de calcium, au contraire, sont insolubles.

\section{L'alcalinisation par la chaux suivie de barbotage carbonique.} - Il est intéressant de savoir ce que l'on obtiendrait avec des laits additionnés de chaux dans lesquels on ferait ultérieurement barboter du gaz carbonique, afin, non seulement de détruire l'alcalinité due à la chaux ajoutée, mais aussi de développer dans la liqueur une acidité due au gaz lui-même après que la chaux aurait été antérieurement neutralisée. La réponse est facile à donner. Lorsqu'à du lait, nous ajoutons de la chaux, puis que nous faisons barboter $\mathrm{CO}^{2}$, dès l'instant où la neutralisation de la chaux est atteinte, l'excès de gaz carbonique tend à former du bicarbonate calcique, c'est-à-dire un sel soluble de calcium dont la présence ne peut que favoriser l'emprésurage.

Nous avons vu un peu plus haut que le simple barbotage carbonique facilite singulièrement la coagulation du lait par l'apport des ions $\mathrm{H}$ qu'il engendre. Mais ici, nous avons commencé par apporter au lait une charge supplémentaire en ions Ca.

Nous reviendrons sur ce point particulier, lorsque nous relaterons les expériences qui ont porté sur les laits additionnés de sels calciques solubles.

INFLUENCE du BICARBONATAGE SUR L'EMPRESURAGE. - Tout à I'heure, nous avons vu les résultats de l'alcalinisation du lait par les bases libres; voyons maintenant ce qui résulte de l'addition des carbonates de ces bases. Nous nous sommes contenté d'étudier l'influence du bicarbonatage du lait sur l'action de la présure, le bicarbonate de sodium étant souvent employé par des commerçants sans conscience pour "rattraper" leurs laits acides.

Nous ferons tout d'abord remarquer que pour abaisser l'acidité du lait attaqué par les ferments lactiques, il faut, moléculairement parlant, de plus grandes quantités de bicarbonate que de base libre. L'acidité dans un milieu comme le lait est bien neutralisée rapidement, tout de suite peut-on dire, par la base libre, mais elle l'est moins aisément par le bicarbonate de la même base. On n'obtient pas, dans l'un et l'autre cas, des systèmes salins identiques.

Des portions de $100 \mathrm{~cm}^{3}$ de lait frais, éerémé toujours, ont été successivement additionnées de bicarbonate de sodium correspondant à 0 gr. $25,0 \mathrm{gr} .50,1 \mathrm{gr}$., 1 gr. 50,2 gr., et 2 gr. 50 par litre. La quantité de bicarbonate à dissoudre est triturée au préalable dans quelques $\mathrm{cm}^{3}$ de lait tiédi, jusqu'à dissolution complète, et on reverse la solution obtenue dans la prise de $100 \mathrm{~cm}^{3}$ qui avait été diminuée de la petite quantité prélevée pour dissoudre le sel.

Il y a lieu de noter que l'acidité originelle qui était de $19^{\circ} \mathrm{D}$. au début n'a été abaissée d'abord qu'à 18 pour finir à 17. C'est qu'ici, redisons-le, il ne s'agit pas de soude libre, mais de son carbonate acide. 
Voici les résultats abtenus traduits sur la courbe jointe :

TABLEAU LII.

ACTION DU BICARBONATE DE SODIUM SUR LA GOAGULATION DU LAIT PAR LA PRÉSURE

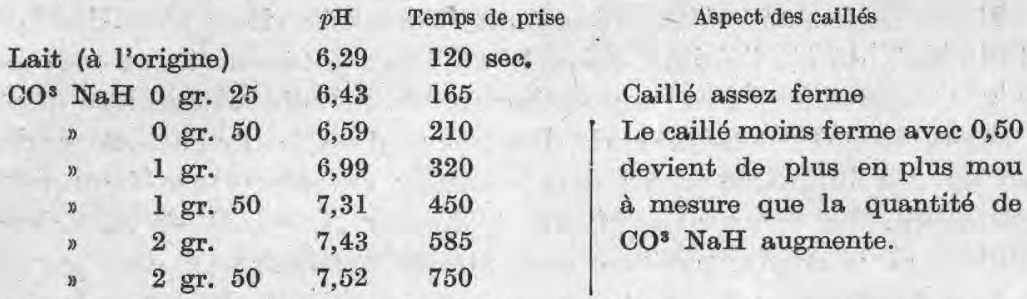

Nous avons cherché à voir ce que deviendrait l'emprésurage après retour des laits bicarbonités vers un? réaction nettement acide.

GRAPHIQUE XVIII.

AGTION DU BIGARBONATE DE SODIUM

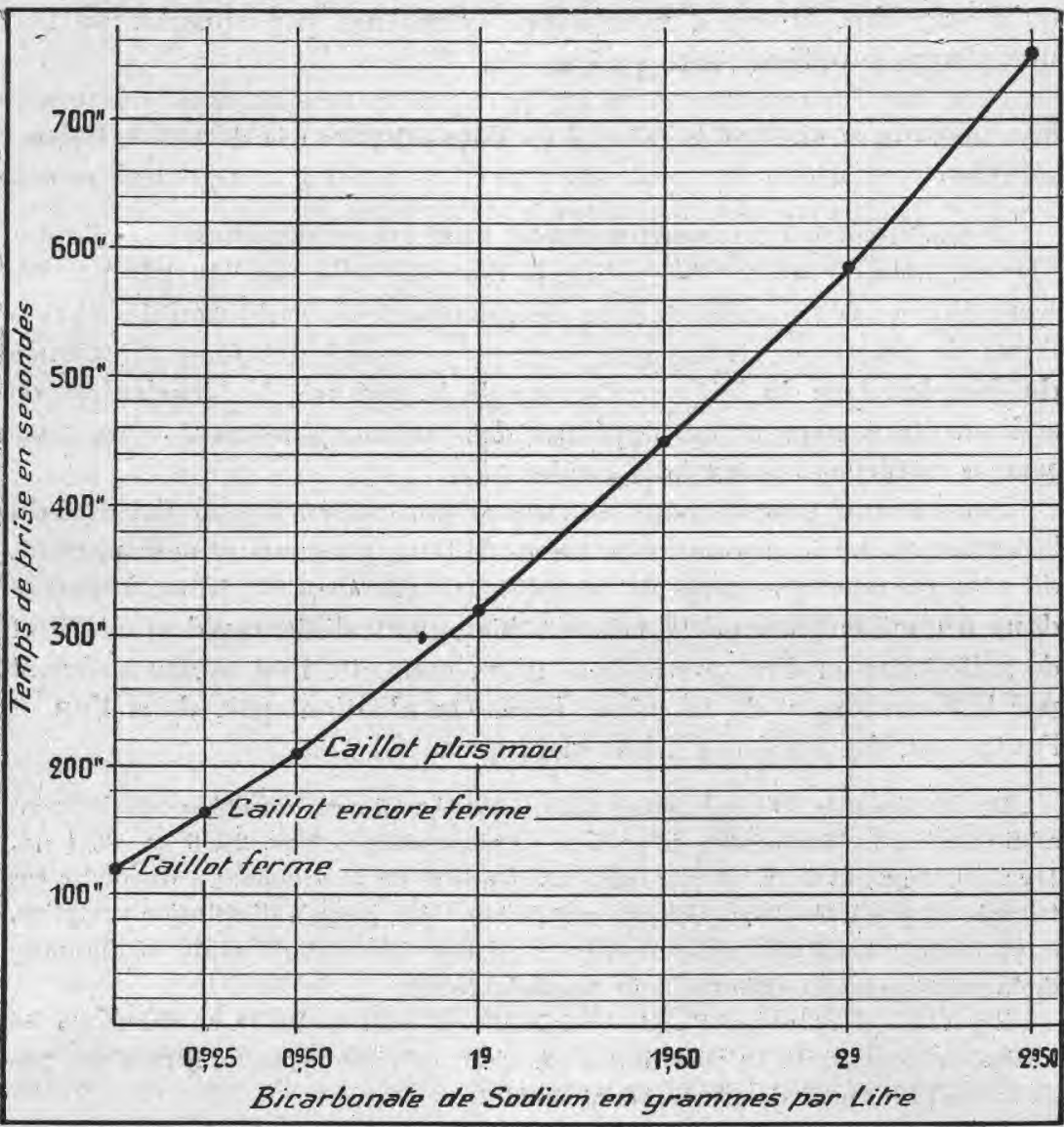


Tous les laits de l'expérience ci-dessus ont été mélangés et $25 \mathrm{~cm}^{3} \mathrm{du}$ mélange font prise à $40^{\circ}$ avec $1 / 2 \mathrm{~cm}^{3}$ de présure en 435 secondes. $100 \mathrm{~cm}^{3}$ de ce mélange sont additionnés d'une assez forte proportion d'acide chlorhydrique, $2 \mathrm{~cm}^{3} \mathrm{HCl} \mathrm{N} / 1$. Il en résulte que l'acidité du lait remonte à $35^{\circ}$ Dornic, c'est-à-dire très fortement; les $100 \mathrm{~cm}^{3}$ ainsi acides font prise en $35 \mathrm{sec}$. seulement, c'est-à-dire en un temps 12,5 fois plus faible que le lait bicarbonaté.

Nous étions guidé dans cette recherche par l'idée que du lait additionné d'eau de Vichy, par exemple, une fois dans l'estomac, soumis par conséquent à l'action de l'acide chlorhydrique du suc gastrique, ferait prise aussi vite que s'il n'avait pas été additionné d'eau minérale, l'acide chlorhydrique ayant neutralisé le bicarbonate sodique.

\section{BIBLIOGRAPHIE ANALYTIQUE}

\section{JOURNAUX, REVUES, SOCIÉTÉS SAVANTES PRODUCTION, HYGIÈNE}

\section{R.-C. FISHER et G.-C. WHITE - Le nettoyage des machines à traire.} Connecticut Storrs Sta. Bul. 144 (1927), 20 p. 6 fig. An in. Bul. mens. Rens. Frig, novembre-décembre 1927, p. 1102.

Le nettoyage et la stérilisation des pièces de caoutchoue des macìines à traire constituent un problème important. On fit des essais avec quatre méthodes de stérilisation. Les agents employés étaient du áésinfectant B K., de l'eau chaude, de la vapeur et de l'eau courante froide. On utilisa chaque méthode pendant une période totale d'environ huit semaines. On fit des dénombrements bactériens sur du lait trait avec des ustensiles dont certaines parties étaient stérilisées de différentes manières ; on établit des statistiques du temps employé à l'entretien des machines, et on fit des observations au sujet de l'action des différents agents sur les pièces de caoutchouc. Après la traite, on fit passer de l'eau froide dans chaque machine, et ensuite de l'eau chaude à travers les tubes. Tout l'outillage fut démonté une fois par semaine et nettoyé à la brosse. Les seaux furent stérilisés quotidiennement à la vapeur.

Comme on l'a constaté précédemment, la solution B. K. à concentration ordinaire est insuffisante pour maintenir à un bas niveau la tenear en bactéries. Une solution à concentration double ( 8 onces pour 10 gallons d'eau) convient si elle est renouvelée deux fois par semaine ou si une surcharge de 4 onces est ajoutée tous les deux jours. La stérilisation ì l'eau chaude à $200^{\circ} \mathrm{F}$. $(3.2 \mathrm{C})$ pendant cinq minutes donne de faibles teneurs en bactéries et le dommage causé aux pièces do caoutchoue n'est pas excessif par cette méthode On ne peut à de plus basse températures compter sur une stérilisation réelle. La stérilisation à la vapeur, tout en réussissant à tuer les bactéries, détruit rapidement le caoutchouc. L'emploi de l'eau froide à moins de $55^{\circ} \mathrm{F}$. $\left(12^{\circ} \mathrm{BC}\right)$ est efficace, mais ce procédé est impropre en été à cause de la température élevée de l'eau. Quel que soit le traitement, l'accumulation bactérienne dans les tubes peut être réduite par rinçage à l'eau froide 